\title{
Emerging Concerns on Air Pollutants from Chemistry Laboratories of University: Ozone Formation Potential, Emission Characteristics, and Emission Inventory in Beijing, China
}

\section{Chenlong Wang}

Beijing Municipal Institute of Labor Protection

Xiaoxi Zhang

Beijing Municipal Institute of Labor Protection

Kun Wang

Beijing Municipal Institute of Labor Protection

Jiajia Gao

Beijing Municipal Institute of Labor Protection

Penglai Zuo

Beijing Municipal Institute of Labor Protection

Quanming Liang

Beijing Municipal Institute of Labor Protection

Yun Zhang

Beijing Municipal Institute of Labor Protection

Yali Tong

Beijing Municipal Institute of Labor Protection

Li Tong

Beijing Municipal Institute of Labor Protection

Yue Han

Beijing Municipal Institute of Labor Protection

Tao Yue ( $\nabla$ dapc@bmilp.com )

Beijing Municipal Institute of Labor Protection

\section{Research Article}

Keywords: urban environment, chemical laboratory, atmospheric pollutants, emission characteristics, emission inventory, ozone formation potential

Posted Date: May 18th, 2021 
DOl: https://doi.org/10.21203/rs.3.rs-449206/v1

License: (c) (1) This work is licensed under a Creative Commons Attribution 4.0 International License. Read Full License 
1 Emerging Concerns on Air Pollutants from Chemistry Laboratories of University: Ozone Formation

2 Potential, Emission Characteristics, and Emission Inventory in Beijing, China

3 Chenlong Wang ${ }^{\text {a }}$, Xiaoxi Zhang ${ }^{\text {a }}$, Kun Wang ${ }^{\text {a }}$, Jiajia Gao ${ }^{\text {a }}$, Penglai Zuo ${ }^{\text {a }}$, Quanming Liang ${ }^{\text {a }}$, Yun Zhang ${ }^{\text {a }}$, Yali

4 Tong ${ }^{\text {a }}$, Li Tong ${ }^{\text {a }}$, Yue Han ${ }^{\text {a }}$, Tao Yue ${ }^{\text {a, * }}$

5 a Beijing Municipal Institute of Labour Protection, Beijing 100054, China.

6 * Corresponding author, Tel: +86010 63524193, Fax: +86 010 63524193. Email Address: dapc@bmilp.com.

\section{$7 \quad$ Acknowledgements}

8 This work was supported by the Fund of Beike Mengya Plan by Beijing Academy of Science and Technology

$9 \quad$ [grant numbers BGS201911].

Abstract: Chemical laboratories of Universities are an important source of air pollutant emissions in urban area, but their detailed emission factors have rarely been investigated. This study determined the concentration level and chemical composition spectrum of air pollutants from 21 typical chemical laboratories of universities in Beijing. Based on quantitative analysis using a GC-MS/FTIR/FID system, the emission intensity of each laboratory area was estimated, the ozone formation potential (OFP) was calculated, and the emission inventory of atmospheric pollutants in chemical laboratories of universities in Beijing was estimated. According to the results, the atmospheric pollutants discharged by the laboratories could be characterized by wide species distribution and outlets were $20.6 \pm 8.9 \mu \mathrm{mol} / \mathrm{mol}$ (mean \pm S.D.), $26.5 \pm 4.8 \mu \mathrm{mol} / \mathrm{mol}$, and $14.7 \pm 5.8 \mu \mathrm{mol} / \mathrm{mol}$. VOC emission was significantly affected using organic solvents. Pollutant emissions from the laboratories exhibited strong periodicity, and the raw materials used in the experiments were the main factor affecting the final pollutant concentration. The emission intensities of atmospheric pollutants from the three outlets were $35.06 \pm 38.08 \mathrm{~g} /\left(\mathrm{m}^{2} \cdot \mathrm{d}\right), 22.83 \pm 18.88$ $\mathrm{g} /\left(\mathrm{m}^{2} \cdot \mathrm{d}\right)$ and $24.03 \pm 28.78 \mathrm{~g} /\left(\mathrm{m}^{2} \cdot \mathrm{d}\right)$, and their TOFP were $27.8 \pm 39.1 \mu \mathrm{mol} / \mathrm{mol}, 22.0 \pm 21.2 \mu \mathrm{mol} / \mathrm{mol}$, and 14.5 $\pm 28.9 \mu \mathrm{mol} / \mathrm{mol}$. The total emission of atmospheric pollutants from university chemical laboratories in Beijing in 2019 was estimated at approximately $2630.8 \pm 2710.3 \mathrm{t}$, including $675.8 \pm 610.6 \mathrm{t}$ of inorganic gaseous pollutants and $1932.0 \pm 2081.2 \mathrm{t}$ of VOCs, with Haidian District as the largest contributor.

Keywords: urban environment; chemical laboratory; atmospheric pollutants; emission characteristics; emission inventory; ozone formation potential

\section{Introduction}

Laboratories are an indispensable workplace for teaching and scientific research in academic institutions. However, they also pose threats to the atmospheric environment due to their emissions. A large number of volatile 
chemical reagents and multi-component compressed gases are used in the working process of chemical laboratories. Moreover, thermophysical experiments such as high-temperature pyrolysis and combustion are performed in some laboratories. During experiments, a large number of harmful gases are generated, which poses great safety hazards. In order to ensure safety, laboratories are equipped with gas collectors, such as fume hood and gas collection hood, to gather toxic and harmful gases and exhaust them into the atmosphere through ventilation ducts.

In recent years, with the rapid development of science and technology in China, multi category and crossdisciplinary laboratories have been established. According to the Beijing Statistical Yearbook 2020, there are 175 colleges and universities in Beijing, 112 of which are involved in the use of laboratories. Such 45 colleges and universities are mainly distributed in the center area in Beijing that contained Haidian, Xicheng, Dongcheng, Chaoyang, Fengtai, Shijingshan what the local people called "the six districts in city". With such a large number and wide variety, laboratories, especially chemical laboratories, will inevitably release pollutants to the urban atmospheric environment. Gas emissions from laboratories have brought a series of environmental problems. The most obvious issue is the odor of the emitted gases, which is a major complaint of residents and households around the laboratory (Xue et al., 2020). After preliminary test, the main components of these exhaust gases are volatile organic compounds (VOCs), including esters, aromatic compounds, polycyclic aromatic hydrocarbons, aldehydes, alcohols, and ketones, which are resistant to degradation. Some VOCs, such as benzene, polycyclic aromatic hydrocarbons (PAHs), and heterocyclic aromatic amine (HCAs), are even carcinogenic to humans (Kabir, 2010; Vicente, 2018). Moreover, some VOCs in laboratory exhaust, such as alkane, toluene, and xylene, react photochemically with nitrogen oxides $\left(\mathrm{NO}_{\mathrm{X}}\right)$ to produce ozone in the atmosphere, and these VOCs are thus called ozone potential bodies. The increase in ozone level due to emissions of these VOCs (Kim and Lee, 2018; Shin et al., 2013; Wang, 2018) will contribute to the formation of photochemical smog and secondary organic aerosols, which are hazardous to human health and the environment (Jiaru, 2020; Qu, 2020). Different VOCs exhibit different reactivities to ozone generation, and the main approach toward analyzing the impact of VOCs on the atmospheric environment is to evaluate VOC reactivity (Robert and Avery, 2006). Therefore, it is crucial to monitor and analyze gas emissions from chemical laboratories.

Although more than 20 industrial air pollutant emission standards have been issued by Ministry of Ecology and Environment of the People's Republic of China (MEE), none of them was limited the pollutant emissions from chemical laboratories. And on May 24, 2019, MEE issued the VOC Unorganized Emission Control Standard (GB37822-2019) to solve the problem of VOC pollution. However, this standard did not clearly define chemical 
laboratories as VOC emission sites for supervision as well. The Regulations of Beijing Municipality on the Prevention and Control of Atmospheric Pollution requires institutions discharging toxic and harmful gases into the atmosphere to install purification devices or take other measures to prevent pollution of the surrounding environment. However, the control and supervision of atmospheric pollution from chemical laboratories are still very weak, and analyses on the composition and emission law of waste gas from chemical laboratories are lacking.

In recent years, some scholars have analyzed characteristic pollutant emissions according to the type of solvent used (Tong et al., 2019; Tian et al., 2017; Fang et al., 2019). Guo et al. (2019) investigated indoor air quality related to chemical lab activities and evaluated the effect of reagent volatilization of indoor air pollutants on the human body. Some scholar attempted to reveal the emission conditions of part of the pollutants in chemical laboratories through various methods, such as the analysis of chemical laboratory exhaust emissions and the introduction of two methods of processing chemical laboratory waste gas (Zhao et al., 2020). Xue et al. (2020) calculated the VOC production and emissions of a chemical laboratory in Beijing using the material balance algorithm; a photoionization ionization detector (PID) was used to detect the concentration of VOC pollutants inside some of the laboratory fume hood, and the VOC emission level of the laboratory was preliminarily identified. Florent et al. (2020) studied the influence of indoor ventilation efficiency on the release of VOCs by substances, and concluded that the ventilation effect would significantly affect the release of individual VOCs. However, the systematic and comprehensive description of the emission characteristics of atmospheric pollutants produced by chemical laboratories have rarely been reported, and specific analyses on the impact of atmospheric pollutants discharged by chemical laboratories on atmospheric environmental quality are lacking.

In order to solve the abovementioned problems, based on long-term and periodic field monitoring, this study determined the emission concentration level and chemical composition spectrum of air pollutants from three typical chemical laboratories in Beijing. The emission factor method was used to calculate the emission inventory of atmospheric pollutants from chemical laboratories of universities in Beijing. Moreover, the impacts of chemical laboratory emissions on the environment were explored. The findings of this study provide scientific basis and technical support for the prevention and control of air pollutants from chemical laboratories of academic institutions.

\section{Materials and methods}

2.1 Monitoring object 


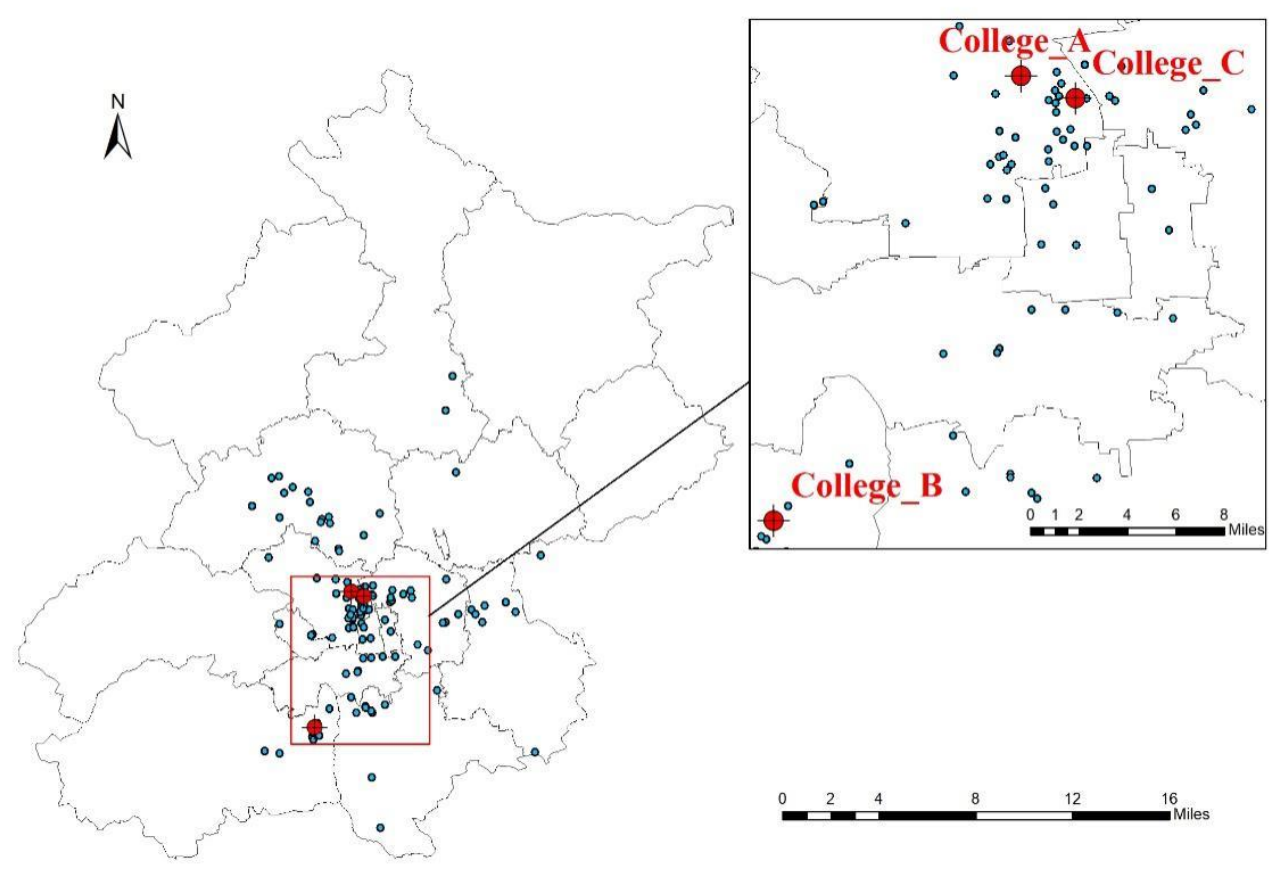

Figure 1 Geographic location of universities in Beijing and the surveyed universities

The laboratory of University A was set with an independent outlet, as the monitoring object, which just connect with a single organic chemistry laboratory. The laboratories of University B and C had comprehensive exhaust outlets, which were connected by an air duct to the exhaust system of several laboratories and sent to the roof for discharge. As the comprehensive outlets are all in series, check valves were installed at the access ports of each laboratory to prevent back-flow. Therefore, there was no air back-flow at the interface, and the sample at the outlet reflects the real emission of each laboratory. The comprehensive outlets of 8 laboratories in the $2-9$ th floors of University B and 12 laboratories in the 1-12th floors of University C were selected. Basic information of corresponding laboratories for each outlet is shown in Table 1.

2.2 Sample collection and analysis

\subsubsection{Analysis of inorganic gaseous pollutants}

The sources of inorganic gaseous pollutants in chemical laboratories mainly include volatilization of volatile reagents such as strong acid and alkali, leakage and emission of inorganic gases such as standard gas and carrier gas, and exhaust gas generated during combustion. In this study, some standard methods, such as HJ688, HJ549, HJ1040, HJ544, and HJ1076, were referred. A gas collector (Laoying ${ }^{\mathrm{TM}}$ 3072, Tsingdao, China), which was 
connected with an acid/alkaline-base absorption liquid, was employed for inorganic gas sampling. All collected samples were tightly sealed and properly stored before further analysis in the laboratory.

The inorganic components in the absorption liquid were preliminarily analyzed using the ICS-1000 ion chromatograph (DIONEX ${ }^{\mathrm{TM}}$, United States). Subsequently, the DX4000 Fourier Transform Infrared spectrometer (FTIR) (Gasmet ${ }^{\mathrm{TM}}$, Finland) was used to conduct periodic continuous monitoring of the preliminarily screened substances. All samples were collected on a pipe connected to a flue-gas concentrated fan.

\subsubsection{Analysis of volatile organic compounds}

VOCs were produced by the volatilization of organic chemical reagents and chemical reactions in the process of reagent mixing. It was collected using a 3.2 L stainless steel Summa tank with external Teflon pipe (Entech ${ }^{\mathrm{TM}}$, USA). The gas collecting head of the pipe was inserted into the center of the flue gas duct for $45 \mathrm{~min}$, and 5 samples were collected every day. Then, the chemical components of VOCs were analyzed by gas chromatography-mass spectrometry (Trace 1300/ISO QD, Thermo ${ }^{\mathrm{TM}}$, USA) with reference to EPA TO15. The components were monitored periodically via FTIR spectroscopy (DX4000, Gasmet ${ }^{\mathrm{TM}}$, Finland) and non-methane hydrocarbons (NMHC), which is the comprehensive index was monitored periodically via a portable flame ionization detector (FID) (Huayi ${ }^{\mathrm{TM}}$, Tianjin). Similar to the continuous monitoring of inorganic gaseous pollutants, VOCs were collected on the pipe of the flue gas outlet.

\subsubsection{Sampling time and quality-checks of the data}

Periodic monitoring of pollutant emissions from the chemical laboratories of 3 universities were conducted from June to October, 2019. Each outlet was monitored for at least 5 days, which from Monday to Friday, and the monitoring time was from 9:00 am to 17:00 pm every day. By removal of outliers such as the data less than the check-out limit, trim the data of unstable time such as before power-on stabilization, $\mathrm{N}_{2}$ gas cleaning time. 4233 values which averaged by minute from FTIR analysis system were obtained.

\subsection{Ozone formation potential (OFP)}

Currently, there are many indicators for evaluating the reactivity of VOCs, and different scholars adopt different methods according to different situations (Chen and Luo, 2012; Zhang et al., 2012; Zeinali et al., 2011; Martien et al., 2003; Carter, 1996). In this study, the maximum incremental reactivity (MIR) method was adopted to characterize the reactivity of VOCs discharged by chemical laboratories and the OFP of the surface boundary layer (Carter, 2010). The MIR coefficient was based on the latest research results of Cater Laboratory in 2013 (Cater, 2013). Equations (1) and (2) were used to analyze the OFP of VOCs.

$$
C_{j}^{M I R}=M I R \times C_{j} \times \frac{\mu_{j}}{\mu_{\text {ozone }}}
$$


where, $C_{j}^{M I R}$ is the maximum ozone concentration that a certain VOC can produce, mol $/ \mathrm{mol} ; M I R$ is the maximum reactivity factor of some VOCs, $\mathrm{gO}_{3} / \mathrm{g} \mathrm{VOCs} ; C_{j}$ is the volume fraction of some VOCs, $\mathrm{mol} / \mathrm{mol} ; \mu_{j}$ is the molecular weight of VOCs, $\mathrm{g} / \mathrm{mol}$;

$\mu_{\text {ozone }}$ is the relative molecular weight of ozone, $48 \mathrm{~g} / \mathrm{mol}$; OFP $P_{M I R}$ is the maximum ozone concentration produced by total VOCs, $\mathrm{mol} / \mathrm{mol}$; and $j$ is the category of VOCs.

\subsection{Emission inventory}

The main equipment for discharging atmospheric pollutants in the laboratories are fume hoods and gas hoods. According to various design specifications, the ventilation system should be installed in direct proportion to the area of the laboratory. In other words, the exhaust flow of air pollutants in the laboratory should be proportional to the usable area of the laboratory. Therefore, the usage area of the laboratory can be used to measure the emission intensity of the laboratory. To evaluate the emission intensity of air pollutants in the chemical laboratory, a pollutant Emission Factor based on the usage area of the chemical laboratory was established as Equation (3).

$$
E M_{i}=\left(\frac{C_{i} \times M_{i}}{V_{m}} \times Q \times t\right) / S \times 10^{-3}
$$

where, $E M_{i}$ is the emission intensity of a single pollutant, $\mathrm{g} /\left(\mathrm{m}^{2} \cdot \mathrm{d}\right) ; C_{i}$ is the measured volume fraction of a pollutant, $\mathrm{mol} / \mathrm{mol} ; M_{i}$ is the molar mass of a pollutant molecule, $\mathrm{g} / \mathrm{mol}$; and $V_{m}$ is the molar volume of the gas, $\mathrm{L} / \mathrm{mol} ; Q$ is the measured fan exhaust air volume, $\mathrm{m}^{3} / \mathrm{h} ; t$ is the operating time of the laboratory day, $\mathrm{h} / \mathrm{d}$; and $S$ is the total usable area of the test laboratory, $\mathrm{m}^{2}$. During the test period, the average temperature and air pressure in Beijing were $25^{\circ} \mathrm{C}$ and $101,000 \mathrm{~Pa}$ (measured by Testo 480 Detector) , $V_{m}=24.5 \mathrm{~L} / \mathrm{mol}$, respectively.

The emission intensity method was used to measure the emission of atmospheric pollutants from laboratories in Beijing. By obtaining the activity level information of university laboratories in Beijing and combining with the relevant emission intensity coefficient, the emission amount of pollutants can be obtained. This method is suitable for regional scale research (Huang et al., 2014; Yan et al., 2017), and the calculation method is shown in Equation (4).

$$
E_{i}=\sum E M_{i} \times A_{t} \times S_{t}
$$

where, $E_{i}$ is the total discharge of a certain pollutant, t/a; $E M_{i}$ is the emission intensity of a single pollutant, $\mathrm{g} /\left(\mathrm{m}^{2} \cdot \mathrm{d}\right) ; A_{t}$ is the average operation days of laboratories of the study area in a year, $(\mathrm{d} / \mathrm{a}) ; S_{t}$ is the total area of the chemical laboratories in the study area, $\mathrm{m}^{2}$.

\section{Results and discussion}




\subsection{Emission characteristics of air pollutants from laboratories}

The analyses revealed 47 types of atmospheric pollutants from the chemical laboratories of the three universities that were stable above the detection limit. These included 8 types of inorganic pollutants, 12 types of alkanes, 1 type of olefins, 8 types of the benzene series (BTEX), 8 types of halogenated hydrocarbons, 8 types of oxygen-containing organic compounds (OVOCs), and 2 types of nitrogenous organic compounds (NVOCs), as shown in Table 2.

Take the sum of the mixing ratios of all measured species, the average concentration of atmospheric pollutant of the three tested units showed a relatively low difference, ranging from 14.65 to $26.47 \mu \mathrm{mol} / \mathrm{mol}$. Among them, University B exhibited the highest overall emission concentration, and its dominant component was nitrogen oxide (12.85 $\mu \mathrm{mol} / \mathrm{mol}, 48.5 \%)$, followed by University A $(6.33 \mu \mathrm{mol} / \mathrm{mol}, 30.7 \%)$. The possible reason is that concentrated nitric acid was used in one laboratory for sample digestion during the test process in University A; in University B, combustion experiments were being conducted in two laboratories and nitric acid was used for digestion in one laboratory, leading to the emission of high concentrations of nitrogen oxides. In terms of VOC emission, the following order was observed: University A $(12.32 \mu \mathrm{mol} / \mathrm{mol})>$ University B $(11.11 \mu \mathrm{mol} / \mathrm{mol})>$ University C (10.24 $\mu \mathrm{mol} / \mathrm{mol})$. Halogenated hydrocarbons (A: 30.5\%) and alkanes (B: 40.8\%, C: 37.7\%) were the dominant components in VOCs. In University A, a large amount of carbon tetrachloride and vinyl chloride were used as experimental solvents, which led to the obvious increase of chlorinated hydrocarbons in exhaust gas. A large amount of petroleum ether was used as solvent in the pharmaceutical engineering laboratory of University B and the organic chemistry laboratory of University C. The test results showed that the content of n-hexane in the exhaust gas VOCs from the laboratories of University B and C accounted for $12.9 \%$ and $14.0 \%$, respectively. These results show that various chemical experiments performed in the laboratories can produce pollutants, which may be emitted to the atmospheric environment. Furthermore, due to of University $\mathrm{C}$, the proportion of halogenated hydrocarbon emissions was high (29.6\%) in University C because of the use of carbon tetrachloride as an organic solvent in its material testing laboratory.

Through the periodic monitoring of air pollutants from the chemical laboratories of three universities, a strong consistency was observed in the time series of the pollutant emissions, which can be divided into three periods: 9:00-12:30 a.m., 12:30-13:30 p.m., and 13:30-17:00 p.m. These periods were determined by the unified work and rest timing of colleges and universities.

In order to study temporal changes of atmospheric pollutant emissions, NMHC, the comprehensive emission index, was selected for time series analysis. All 13 days sampling data of the 3 universities were sorted according 
to the period 9:00-17:00 every day, and fitting statistics were conducted at the same time point of each day, as shown in Figure 2. The graph corresponding to each time point is composed of the normal distribution of the 13day data, the blue solid line represents the average of the 13-day data, and the shaded part represents the $95 \%$ confidence interval of the corresponding data at each time point. NMHC emission was characterized by intermittent low peak and low mean value, with 3-6 emission peaks appearing in a day. The maximum emission concentration during the sampling period was $11.98 \mathrm{mg} \mathrm{C} / \mathrm{m}^{3}$, but the peak occurred only for a short time, and the concentration tended to be flat after approximately $10 \mathrm{~min}$. This characteristic was closely related to a large number of teaching experiments in universities. As we all know, a great feature of teaching experiment is unsustainable for a long time. In general, the discharge of laboratory pollutants was significantly influenced by the working conditions of the laboratory, and was highly instantaneous.

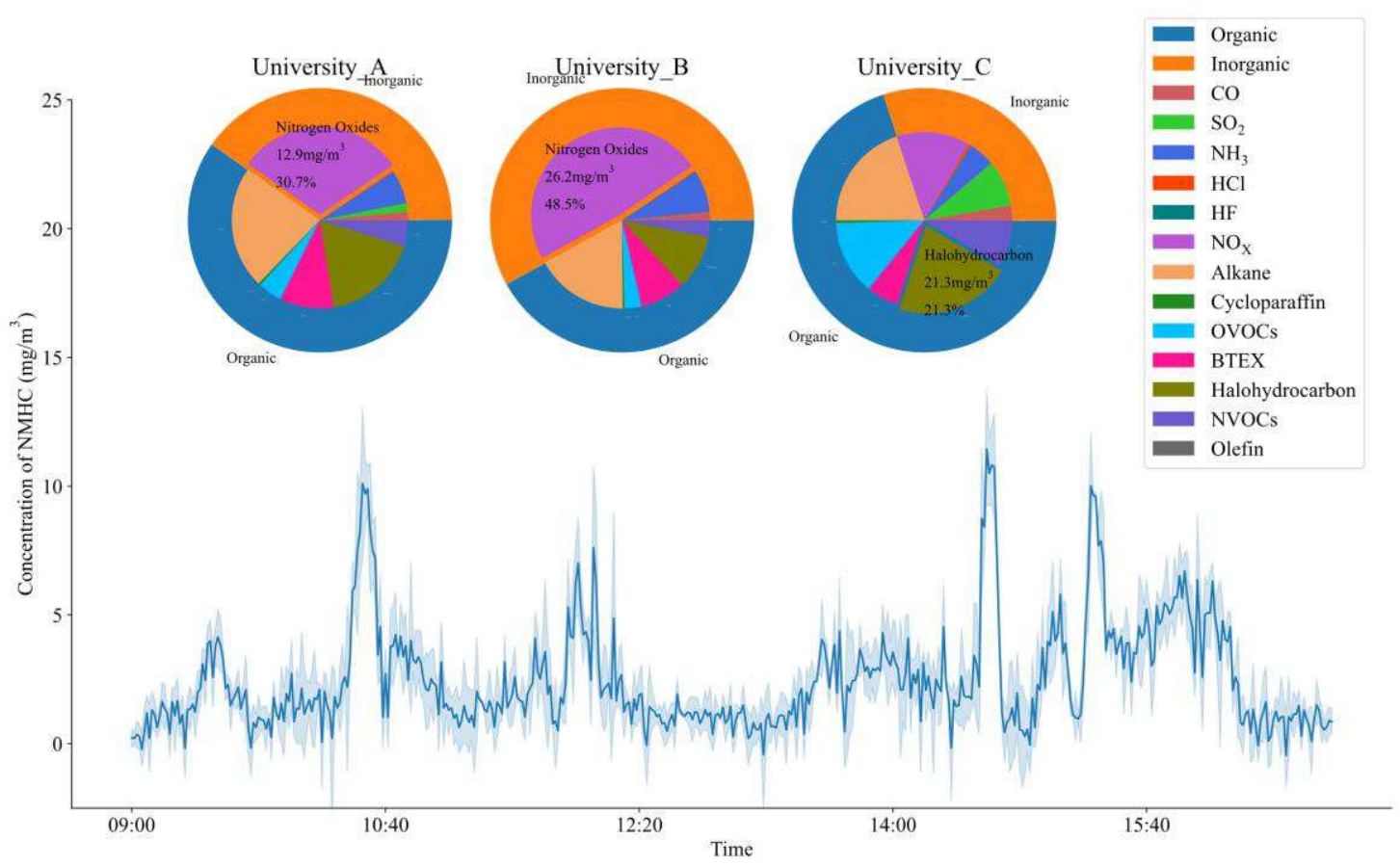

Figure 2 Emission concentration and distribution of atmospheric pollutants in 3 laboratories

\subsection{Ozone formation potential}

According to Equations (2) and (3), the $C_{j}^{M I R}$ values of dominant VOC species and the $O F P_{M I R}$ values of the VOCs discharged from different units of the laboratories are shown in Table 3.

Ethylbenzene, o-xylene, n-hexane, and formaldehyde were the largest contributors to OFP, accounting for $52.0 \%-79.7 \%$ of the total OFP (TOFP). For VOC species with low reactivity, even if their concentration is high, they will not significantly contribute to ozone formation. On the contrary, even low concentrations of highly reactive VOCs species may contribute significantly to ozone production (Tang et al., 2016). Combined with Figure 

hydrocarbons have a greater impact on the atmospheric environment owing to their high MIR values. Therefore, the control of low concentration and highly active substances is a key aspect of laboratory pollutant control.

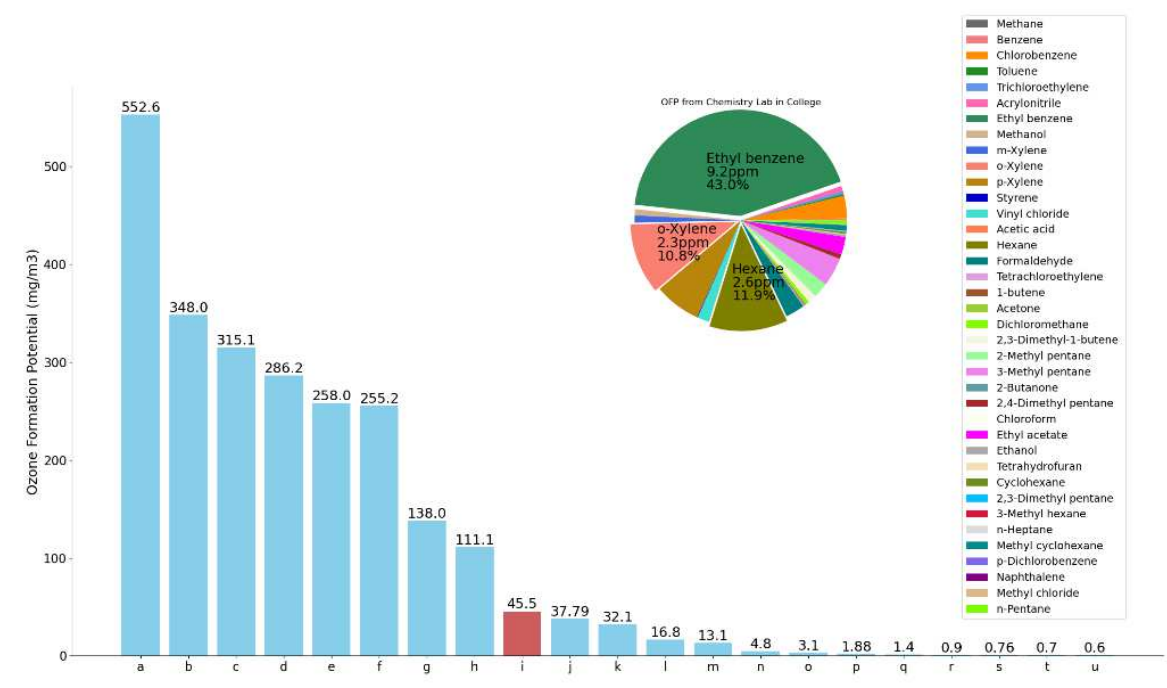

219

*a: Coke oven flue (Zhang et al., 2020), b: furniture (Zhang et al., 2019), c: medical (Li et al., 2014), d: furniture (Zhang et al., 2019), e: furniture (Zhang et al., 2019), f: medical (Li et al., 2014), g: furniture (Zhang et al., 2019), h: medical (Li et al., 2014), i: chemistry lab, j: medical (Li et al., 2014), k: medical (Li et al., 2014), 1: coking plant (Zhang et al., 2020), m: coking plant (Zhang et al., 2020), n: coking plant (Zhang et al., 2020), o: medical (Li et al., 2014), p: printing (Liu et al., 2019), q: plywood (Lv et al., 2020), r: printing (Liu et al., 2019), s: iron and steel(Zhang et al., 2020), t: plywood (Lv et al., 2020), u: plywood (Lv et al., 2020)

Figure 3 OFP in different studies (Li et al., 2014; Liu et al., 2019; Lu et al., 2020; Xu et al., 2020; Zhang et al., 2019; Zhang et al., 2020)

The ozone generating potential of VOCs emitted by the chemical laboratory in this study was compared with that of other typical VOC emission sources, as shown in Figure 3. It was found that compared with other research, the influences of different industries and different solvent processes on TOFP were significantly different. (Li et al. 2014) conducted a study on the chemical synthetic pharmaceutical industry, and found that the TOFP values of VOCs emitted from the exhaust were significantly different, ranging from 3.1 to $315.1 \mathrm{mg} / \mathrm{m}^{3}$, which could be attributed to differences in the raw materials and excipients used by various enterprises and the emission concentration. (Lu et al. 2020) studied a typical plywood manufacturing enterprise and found that the contribution of VOCs to TOFP and each component of VOCs to OFP was different in different process stages (hot pressing process, gluing process and exhaust outlet), but the overall TOFP was low, ranging from 0.5 to $1.3 \mathrm{mg} / \mathrm{m}^{3}$. In this study, the OFP of emissions from the laboratories was lower than that of coking plants, pharmaceutical industries, 
and furniture enterprises, but much higher than that of steel works, plywood manufacturing enterprises, packaging

239 printing and publication printing industries. Therefore, the control of atmospheric pollutant emissions, especially

240 VOCs, from chemical laboratories of academic institutions should be strengthened.

$241 \quad 3.3$ Emission inventory of air pollutants from chemical laboratories of universities in Beijing

$242 \quad$ 3.3.1 Emission factor by testing

243 According to Equation (3), the emission intensity of air pollutants in each chemistry laboratory was calculated, and the results are shown in Table 4.

The overall emission intensity of University A was higher than those of the other two universities, with benzene series, halogenated hydrocarbons, and alkanes accounting for a large proportion. The emission intensity of University A was relatively high because the detection point was an organic chemistry laboratory, and the experiments mainly involve organic chemical reagents. The average emission intensity of air pollutants in the three universities was $27.3 \pm 28.6 \mathrm{~g} /\left(\mathrm{m}^{2} \cdot \mathrm{d}\right)$.

\subsubsection{Emission inventory}

According to the National Institution of Higher Education statistics (Wu and Gao, 2014), in 2012/2013, the area of university laboratories in Beijing was 1.8 million $\mathrm{m}^{2}$, with an average annual growth rate of $8.83 \%$. Among them, engineering and science laboratories accounted for $62.59 \%$ of the total number of laboratories. The allocation of laboratory area and emissions was carried out with the number of students in each university as the weight. Combining these statistics with the emissions intensity obtained in this study, an air pollutant emission inventory of chemical laboratories of universities in Beijing in 2019 was established. In 2019, the total emission of atmospheric pollutants from chemical laboratories of universities in Beijing was 2630 tons, including 698 tons of inorganic gaseous pollutants and 1932 tons of VOCs. The corresponding pollutant emissions of chemical laboratories of universities in various districts and counties of Beijing are shown in Table 5.

Haidian district was the main source of atmospheric pollutants emitted by chemical laboratories of universities in Beijing, with the highest total emission of pollutants (2630.8 \pm 2710.3 tons/year) among all the districts of Beijing. This can be explained by Haidian district having the largest number of universities, with a total of 33 , accounting for $28.2 \%$ of the total number of universities in Beijing. According to the development needs of Beijing, colleges and universities gradually set up new sites in the outer suburbs. Combined with Figure 1, With the increasing number of branch campuses in Changping (20 colleges) and Fangshan (10 colleges), the pollutant emissions of chemical laboratories in these two areas have also reached 335.7 tons/year and 225.9 tons/year, ranking the third and fourth among all districts. 

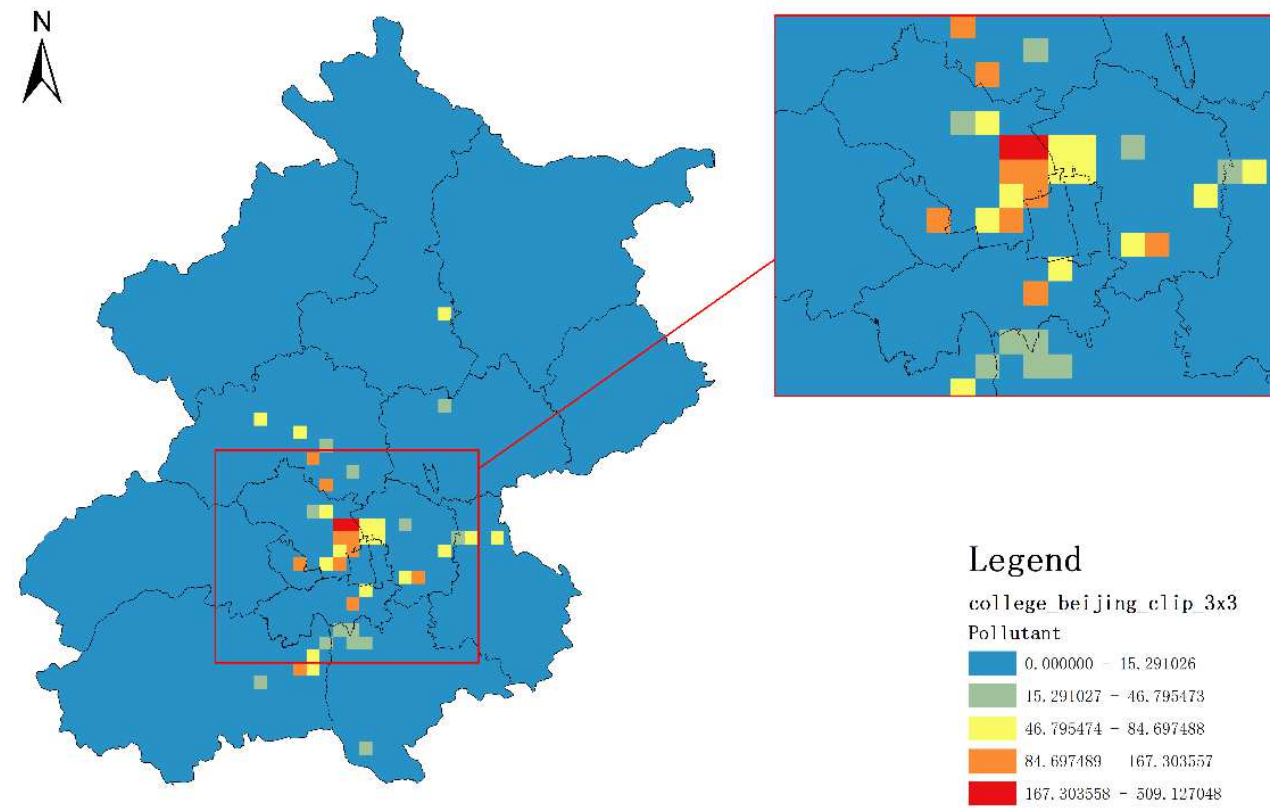

Figure 4 Pollutant emission grid of chemical laboratories of universities in Beijing

As shown in Figure 4, the emission of atmospheric pollutants from chemical laboratories of universities in Beijing was concentrated in the central urban area of Beijing, and the highest pollutant emission was from the southeast of Haidian District, where located lots of universities such as Tsinghua University and Peking University. The distribution was closely related to the development of economy and science and technology in Beijing. The total emission of atmospheric pollutants was within the range of 81-309 tons/grid.

In the figure, the area with high pollutant emission corresponds to the science and technology innovation and development center of Beijing, where universities are more densely distributed and more well-known universities are located. Universities in this area have more abundant means for scientific research than other universities. These universities also use the largest amounts of chemical reagents and emit the largest amount of waste liquid among all the universities in Beijing. 

the 4th among the 17 districts in the city. VOCs emitted by chemical laboratories of universities significantly affect the surrounding residents.

\subsubsection{Uncertainty analysis}

In the estimation of any emission inventory, uncertainties are inevitable because of inadequate or incomplete information and data. These uncertainties might affect the estimation of emission inventories to some extent (Zhong et al., 2007; Wei et al., 2011). In this study, the number of college students was used to allocate the area proportion of chemical laboratories in each university, and measured data from the three universities were used to represent the emission factors of chemical laboratories in all universities, which would lead to certain uncertainty. To assess the uncertainties in the emission inventory, a quantitative approach was applied to estimate the uncertainty range for each class of pollutant using Monte Carlo simulations at $90 \%$ confidence interval. The activity level information was obtained from the survey and annual statistical report, and the uncertainty of activity level information was set as $\pm 80 \%$ with reference to the empirical value of the TRACE-P list (Streets et al., 2003). The uncertainty of the pollutant discharge inventory of chemical laboratories of universities is shown in Table 6 .

\section{Conclusion}

According to the analysis results of this study, 8 types of inorganic substances such as acid inorganic gas and alkaline inorganic gas, and 39 types of organic pollutants such as alkanes, alkenes, benzene series, halogenated hydrocarbons, oxygen-containing organic compounds and nitrogen-containing organic compounds were identified, which were stable above the detection limit of the equipment. Among the three universities, University B showed the highest overall emission concentration of $31.16 \mu \mathrm{mol} / \mathrm{mol}$, with nitrogen oxides as the main component. University A showed the highest VOC concentration $(12.3 \mu \mathrm{mol} / \mathrm{mol})$. In addition, pollutant emissions from the chemical laboratories exhibited strong periodicity, and the periodicity of the same type of laboratory was relatively consistent, which is closely related to the working status and rest time. Pollutant emissions from the laboratories were characterized by intermittent low peak and low mean value, but the peaks existed only for a short time, and the concentration tended to be flat after approximately $10 \mathrm{~min}$. The raw materials used in the laboratory experiments were the main factor determining the final pollutant emission concentration. The emission intensities of air pollutants from the chemical laboratories of the three universities were $35.06 \pm$ $38.08 \mathrm{~g} /\left(\mathrm{m}^{2} \cdot \mathrm{d}\right), 22.83 \pm 18.88 \mathrm{~g} /\left(\mathrm{m}^{2} \cdot \mathrm{d}\right)$ and $24.03 \pm 28.78 \mathrm{~g} /\left(\mathrm{m}^{2} \cdot \mathrm{d}\right)$, and the OFPs were $27.8 \pm 39.1,22.0 \pm 21.2$ and $14.5 \pm 28.9$, which are higher than those of plywood manufacturing enterprises, and packaging and printing industries. In 2019, the total emission of atmospheric pollutants from chemical laboratories of universities in 
314 Beijing was $2630 \mathrm{t}$, including $698 \mathrm{t}$ of inorganic gaseous pollutants and $1932 \mathrm{t}$ of VOCs. Haidian District was the

315 largest contributor to the emissions among all districts of Beijing. The control of low concentration and high

316 activity substances is a key aspect of air pollutant control for chemical laboratories.

\section{Declarations}

\section{Ethics approval and consent to participate}

319 Not applicable

\section{Consent for publication}

$321 \quad$ Not applicable

\section{Availability of data and materials}

323 All data generated or analysed during this study are included in this published article [and its supplementary 324 information files].

\section{$325 \quad$ Competing interests}

326 The authors declare that they have no competing interests

\section{$327 \quad$ Funding} [grant numbers BGS201911].

\section{$330 \quad$ Authors' contributions}

331 CW performed the analysis of testing data, and was a major contributor in writing the manuscript.

332 XZ has contributed a lot to the on-site sampling work.

333 KW calculated the emissions inventory of universities in Beijing.

334 PZ provided ideas for data analysis.

335 JG and YZ translation the manuscript to English language.

336 YT offered suggestions for revision of this manuscript.

337 QL and LT spent a lot of time on site sampling work.

338 YH sorted out and counted the sampling data of the site.

339 TY provided the overall framework of the manuscript.

340 All authors read and approved the final manuscript.

\section{$341 \quad$ References}

342 Beijing bureau of statistics, 2020. Beijing statistical yearbook. http://nj.tjj.beijing.gov.cn/nj/main/2020tjnj/zk/indexch.htm 
Carter, W.P.L., 1996. Development of ozone reactivity scales for volatile organic com- pounds. J. Air Waste Management. Assoc. 44 (7), 881-899.

Carter, W.P.L., 2010. Development of the saprc-07 chemical mechanism. Atmos. Environ. 44 (40), 5324-5335.

Cater,W.P.L., 2013. SAPRC Atmospheric Chemical Mechanisms and VOC Reactivity Scales. https://intra.engr.ucr.edu/ carter/SAPRC/scales07.xls

Chen, J.J., Luo, D.M., 2012. Ozone formation potentials of organic compounds from different emission sources in the south coast air basin of California. Atmos. Environ. 5 (5), 448-455.

Fang L., Liu W.W., Chen D.N., Li G.H., Wang D., Shao X., Nie L., 2019. VOCs composition Spectrum of typical Solvent application industry in Beijing. Environ. Sci. 40(10), 4395-4403. (in Chinese)

Florent C., Romain G., Laurence R., Marie V., Frédéric T, 2020. Behavior of individual VOCs in indoor environments: How ventilation affects emission from materials. Atmos. Environ. S1352-2310 (20), 30445-3

GB 37822-2019. Organic-Free Emission Control Standard for Volatile Organic Compounds.

Guo Y, 2009. Environmental pollution prevention and control in chemical laboratory. Environ. Sci. Technol. 22(S1), 55-57. (in Chinese)

HG/T 20698-2009. Specification for design of chemical heating ventilation and air conditioning.

HJ 1040-2019. Stationary pollution source waste gas - Determination of Hydrogen bromide - Ion chromatography.

HJ 1076-2019. Ambient air - Determination of ammonia, methylamine, dimethylamine and trimethylamine - Ion chromatography.

HJ 544-2016. Determination of sulfuric acid mist in stationary pollution source waste gas - Ion chromatography. HJ 549-2016. Ambient air and waste gas - Determination of hydrogen chloride - Ion chromatography.

HJ 688-2019. Stationary pollution source - Determination of hydrogen fluoride - Ion chromatography.

Hou M.T., Chi N.C., Wang Y.J., Li F.B., Zhang J., Zhang R.Y., 2020. Brief Analysis of VOCs pollution status and Countermeasures in A laboratory. Proceedings of the first session of the national inspection group on post competence improvement of inspection and testing personnel. China Building Materials Science and Technology Magazine, 3. (in Chinese)

Huang Y., Shen H., Chen H., Wang R., Zhang Y., Su S., Chen Y., Lin N., Zhuo S., Zhong Q., Wang X., Liu J., Li B., Liu W., Tao S., 2014. Quantification of global primary emissions of PM2.5, PM10, and TSP from combustion and industrial process sources. Environ. Sci. Technol. 48(23), 13834-13843

Jiaru L., Yosuke S., Nanase K., Tomihide F., Kohei M., Marina T., Jun Z., Maho N., Kentaro M., Yasuhiro S., Yoshihiro N., Kei S., Akinori T., Ayako Y., Tomoki N., Shungo K., Yoshizumi K., 2020. Total hydroxyl 
radical reactivity measurements in a suburban area during AQUAS-Tsukuba campaign in summer 2017. Sci. Total Environ. 740, 139897.

Kabir, E., Kim, K.H., Ahn, J.W., Hong, O.F., Sohn, J.R., 2010. Barbecue charcoal combustion as a potential source of aromatic volatile organic compounds and carbonyls. J. Hazard Mater. 174, 492-499.

Kim, Y.P., Lee, G., 2018. Trend of air quality in Seoul: policy and science. Aerosol Air Qual. Res. 18, 2141-2156.

Li Y., Wang Z.M., Song S., Xu Z.R., Xu M.Z., Xu W.W., 2014. Characteristics and Hazard Assessment of process exhaust VOCs emission in chemical synthetic pharmaceutical industry. Environ. Sci. 35(10), 3663-3668. (in Chinese)

Liang Q., Bao X., Sun Q., Zhang Q.L., Zou X., Huang C.Q., Shen C.Y., Chu Y.N., 2020. Imaging VOC distribution in cities and tracing VOC emission sources with a novel mobile proton transfer reaction mass spectrometer. Environ. Pollut. 265, 114628.

Liu W.W., Fang L., Guo X.R., Nie L., Wang M.Y., 2019. VOCs emission Characteristics and Ozone generation Potential analysis of typical printing enterprises in Beijing-Tianjin-Hebei Region. Environ. Sci. 40(09): 39423948. (in Chinese)

Lv D.Q., Lu S.H., Shao M., Wang L.F., Ren J., 2020. VOCs emission characteristics of typical plywood manufacturing enterprises. Environ. Sci. in China, 40(05):1924-1931. (in Chinese)

Martien, P.T., Harley, R.A., Milford, J.B., Russell, A.G., 2003. Evaluation of incremental reactivity and its uncertainty in southern California. Environ. Sci. Tech. 37 (8), 1598-1608.

Robert J, Avery, 2006. Reactivity-based VOC control for solvent products: more efficient ozone reduction strategies. Environ. Sci. Technol. 40, 4845-4850.

SH/T 3103-2009. Specification for laboratory design of petrochemical Center. Shanghai Engineering Co., LTD.

Sinopec. Chemical Process Design Manual (fourth Edition) [M]. Beijing: Chemical Industry Press, 2009.

Shin, H.J., Kim, J.C., Lee, S.J., Kim, Y.P., 2013. Evaluation of the optimum volatile organic compounds control strategy considering the formation of ozone and secondary organic aerosol in Seoul, Korea. Environ. Sci. Pollut. Res. 20, 1468-1481.

Streets, D.G., Bond, T.C., Carmichael, G.R., Fernandes, S.D., Fu, Q., He, D., Klimont, Z., Nelson, S.M., Tsai, N.Y., Wang, M.Q., Woo, J.H., Yarber, K.F., 2003. An inventory of gaseous and primary aerosol emissions in Asia in the year 2000. J. Geophys. Res-Atmos. 108, 8809.

Tang X.Y., Zhang Y.H., Shao M., 2006. Atmospheric Chemistry. Higher Education Press, Beijing, China . 
Tian L., Wei W., Cheng S.Y., Li G.H., Wang G., 2017. Composition spectrum of VOCs and ozone generation potential in typical organic solvent application industry. Journal of safety and environment. 17(01), 314-320. (in Chinese)

Tong R.P., Zhang L., Yang, X.Y., Liu, J.F., Zhou, P.N., Li, J.F., 2019. Emission characteristics and probabilistic health risk of volatile organic compounds from solvents in wooden furniture manufacturing. J. Clean. Prod. 208, 1096-1108

Vicente, E.D., Vicente, A., Evtyugina, M., Carvalho, R., Tarelho, L.A.C., Oduber, F.I., Alves, C., 2018. Particulate and gaseous emissions from charcoal combustion in barbecue grills. Fuel Process. Technol. 176, 296-306.

Wang, H., Xiang, Z., Wang, L., Jing, S., Lou, S., Tao, S., Liu, J., Yu, M., Li, L., Lin, L., Chen, Y., Wiedensohler, A., Chen, C., 2018. Emissions of volatile organic compounds (VOCs) from cooking and their speciation: a case study for Shanghai with implications for China. Sci. Total Environ. 621, 1300-1309.

Wei, W., Wang, S., Hao, J., 2011. Uncertainty Analysis of Emission Inventory for Volatile Organic Compounds from Anthropogenic Sources in China. Environ. Sci. 32, 305-312 (in Chinese).

Wu X.F., Gao X.J., 2014. University Laboratory Construction and Development Report (2014). Beijing: Tsinghua University Press. 26 34 (in Chinese)

Xue Y.F., Zhang Q.Y., Nie L, Zeng J.H., Zhuang Z.W., GAO X.C., AI D.S., Zhang S.H., Xu K.L., 2020. VOCs emission level and Control countermeasures in Beijing Laboratory. Experimental technology and management, 37(02), 10-14. (in Chinese)

Yan D.J., Su H., Huang X.M., Song W.B., Wang H.Q., Song X.J., 2017. Anthropogenic Volatile organic compounds Emission Inventory and Research in Xi 'an city. Journal of environmental science, 37(02), 446452. (in Chinese)

Zeinali, M., Mcconnell, L.L., Hapeman, C.J., Schmidt, A.N., Howard, W.E., Cody, J., 2011. Volatile organic compounds in pesticide formulations: methods to estimate ozone formation potential. Atmos. Environ. 45 (14), 2404-2412.

Zhang J.N., Zeng C.L., Liu R.Y., Yao Y.J., Ke Y.T., Fan L.Y., Ye D.Q., 2019. Emission Characteristics of Volatile organic compounds in furniture enterprises and its Environmental impact. Environmental science, 40(12):5240-5249. (in Chinese)

Zhang X.F., Gao S., Fu Q.Y., Han D.M., Chen X.J., Fu S., Huang X.Q., Cheng J.P., 2020. Impact of VOCs emission from iron and steel industry on regional $\mathrm{O}_{3}$ and PM2.5 pollutions. Environ. Sci. Pollut. R. 23, 28853-28866. 
Zhang X.M., Wang D., Liu Y., Cui Y.F., Xue Z.G., Gao Z.F., Du J.H., 2020. Characteristics and ozone formation potential of volatile organic compounds in emissions from a typical Chinese coking plant. J. Environ. Sci. 9, 183-189.

Zhang, X.M., Chai, F.H., Yue, T.T., Zhang, K., Chen, Y.Z., Duan, J.C., Xue, Z.G., 2012. Photochemical characteristics and sources of volatile organic compounds in Wuqing, Tianjin. Res. Environ. Sci. 25 (10), $1085-1091$.

Zhao X.D., 2020. Research on waste gas treatment in Chemical Laboratory. Contemporary Chemical Research, 12, 114-115. (in Chinese)

Zhong, L., Zheng, J., Lei, G., Chen, J., 2007. Quantitative uncertainty analysis in air pollutant emission inventories: methodology and case Study. Research of environmental science. 20, 15-20 (in Chinese). 


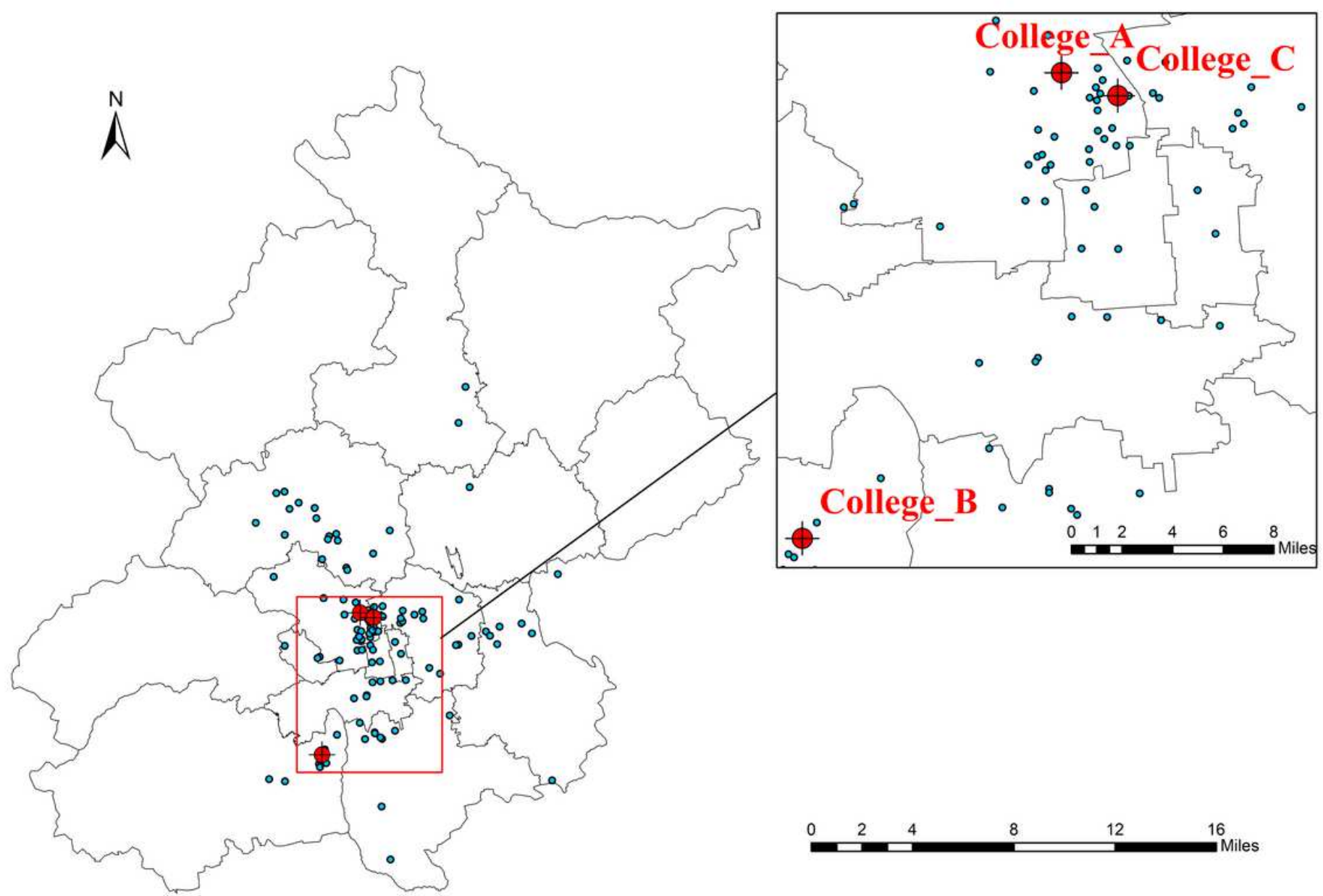

Figure 1

Geographic location of universities in Beijing and the surveyed universities Note: The designations employed and the presentation of the material on this map do not imply the expression of any opinion whatsoever on the part of Research Square concerning the legal status of any country, territory, city or area or of its authorities, or concerning the delimitation of its frontiers or boundaries. This map has been provided by the authors. 


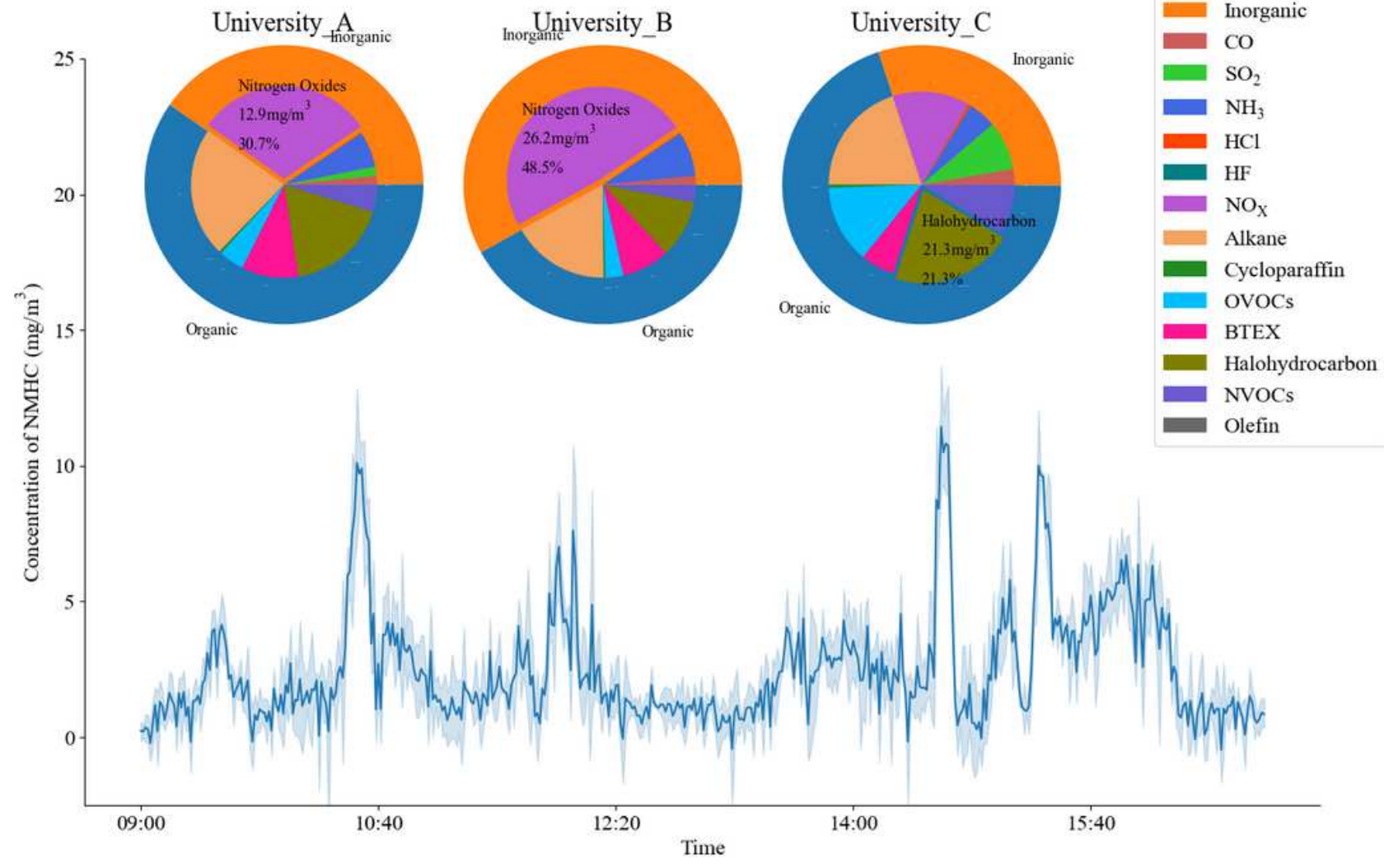

Figure 2

Emission concentration and distribution of atmospheric pollutants in 3 laboratories

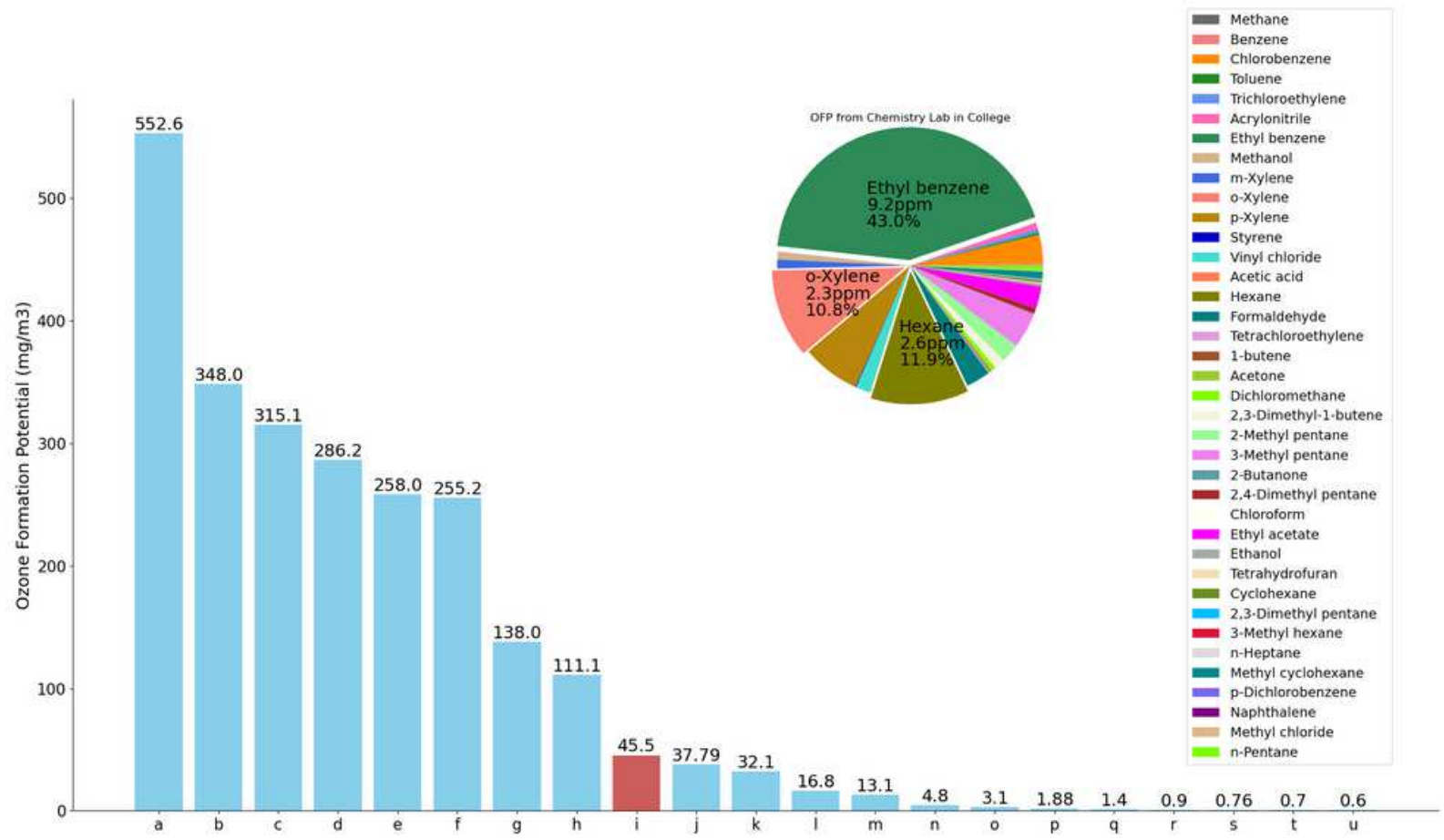




\section{Figure 3}

OFP in different studies (Li et al., 2014; Liu et al., 2019; Lu et al., 2020; Xu et al., 2020; Zhang et al., 2019; Zhang et al., 2020) *a: Coke oven flue (Zhang et al., 2020), b: furniture (Zhang et al., 2019), c: medical (Li et al., 2014), d: furniture (Zhang et al., 2019), e: furniture (Zhang et al., 2019), f: medical (Li et al., 2014), g: furniture (Zhang et al., 2019), h: medical (Li et al., 2014), i: chemistry lab, j: medical (Li et al., 2014), k: medical (Li et al., 2014), l: coking plant (Zhang et al., 2020), m: coking plant (Zhang et al., 2020), n: coking plant (Zhang et al., 2020), o: medical (Li et al., 2014), p: printing (Liu et al., 2019), q: plywood (Lv et al., 2020), r: printing (Liu et al., 2019), s: iron and steel(Zhang et al., 2020), t: plywood (Lv et al., 2020), u: plywood (Lv et al., 2020)
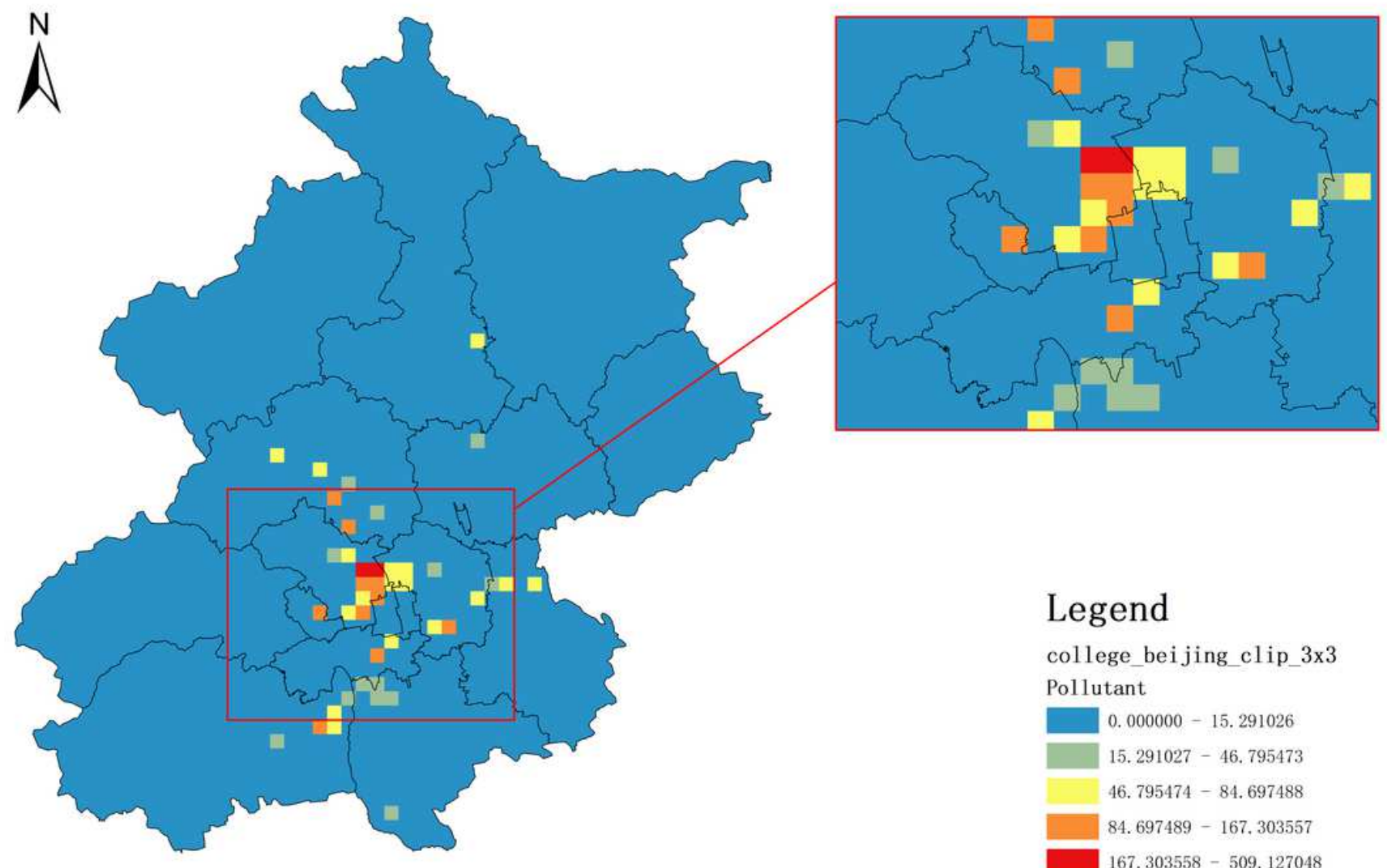

\section{Legend}

college_beijing_clip_3x3 Pollutant

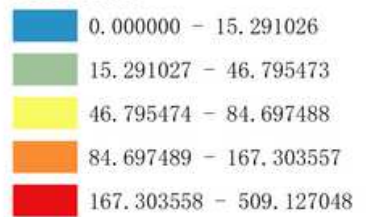

\section{Figure 4}

Pollutant emission grid of chemical laboratories of universities in Beijing Note: The designations employed and the presentation of the material on this map do not imply the expression of any opinion whatsoever on the part of Research Square concerning the legal status of any country, territory, city or area or of its authorities, or concerning the delimitation of its frontiers or boundaries. This map has been provided by the authors. 